\title{
Author Index for Volume 14
}

Ali, F.

Alonso Álvarez, J.N.

Alsheik Ahmad, A.Y.

Arvasi, Z.

Asadollahi, J.

Ayık, H.

Bao, X.M.

Barile, M.

Campbell, C.M.

Cao, W.

Carvalho, C.A.

Chajda, I.

Chang, H.X.

Chen, C.

Chen, H.Y.

Chen, J.L.

Chen, X.W.

Choi, S.H.

Cuong, N.T.

Danchev, P.V.

Denecke, K.

Dibaei, M.T.

Dowbor, P.

Du, Y.

Dung, N.T.

Feng, Y.-Q.

Fernández Vilaboa, J.M.
135

229

25

669

155

279

443

631

279

177

389

191

555

425

613

537

301

479

265

515

191

209, 497

361

301

265,455

351

229
Godloza, L.

González Rodríguez, R.

Goze, M.

Grechkoseeva, M.A.

Groenewald, N.J.

Guo, X.J.

Guo, X.Y.

Haghany, A.

Hajduk, A.

Harris, M.E.

Hayat, U.

$\mathrm{Hu}, \mathrm{J}$.

Jain, S.

Jaraden, J.J.

Jiang, C.P.

Jiang, Q.F.

Jin, Q.Q.

Kehayopulu, N.

Kim, H.

Kim, M.O.

Koşan, M.T.

Li, H.S.

Li, X.L.

Lian, H.F.

Liang, K.

Lin, C.Y.

Loukaki, M.
1

229

313

585

1

$285, \quad 687$

605

489

361

143

97

571

341

25

117

117

521

705

469

469

53

541

37

425

15

555

207 
Lu, X.M.

403

Luo, Y.F.

Ma, Z.Z.

Mao, L.X.

Marczak, A.W.

Moretó, A.

Mushtaq, Q.

Naghipour, R.

Nam, K.-B.

Nhan, L.T.

Obul, A.

O'Connor, J.J.

Olivier, W.A.

Orhan, N.

Ouyang, B.Y.

Park, Y.S.

Płonka, J.

Qin, H.R.

Remm, E.

Ren, C.C.

Ren, X.M.

Rodríguez Raposo, A.B.

Romaguera, S.

Ruškuc, N.

Salarian, Sh.

Samman, M.

Sanchis, M.

Sanwong, J.

Schenzel, P.

Shi, W.J.

Shin, Y.S.

Shoji, K.
593

207

97

505

479

265

639

279

1

625

85

469

593

403

313

285

215

229

167

389

155

79

167

255

505

585

649

245
Shum, K.P. $\quad$ 215, 285, 341, 687

Skiba, A.N. 25

Sullivan, R.P.

255

Tan, S.B.

713

Tong, J.

521

Tong, W.T.

85

Tribak, R.

625

Tsingelis, M.

705

Tütüncü, D.K.

625

Ulualan, E.

669

Vasilev, A.V.

585

Vedadi, M.R.

489

Wang, J.X.

605

Wang, M.-O.

295

Wang, Q(iang)

103

Wang, Q(ing)

713

Wang, Q.W.

555

Wang, X.F.

443

Wang, Y.L.

661

Wang, Z.

537

Wen, Q.Z.

425

Wendt, G.

417

Wismath, S.L.

103, 191

Xia, J.G.

181

Xie, W.W.

443

$\mathrm{Xu}, \mathrm{K} . J$.

661

Yassemi, S.

209, 497

Ye, Y.

301

Zhang, Y.H.

571

Zhou, C.X.

351

Zhou, L.R.

85

Zhu, F.H. 